\title{
An inflammation-related nomogram for predicting the survival of patients with non-small cell lung cancer after pulmonary lobectomy
}

Ying Wang ${ }^{1,4 \dagger}$, Xiao Qu ${ }^{1 \dagger}$, Ngar-Woon Kam ${ }^{4}$, Kai Wang ${ }^{1}$, Hongchang Shen ${ }^{3}$, Qi Liu ${ }^{1 *}$ and Jiajun Du ${ }^{1,2^{*}}$

\begin{abstract}
Background: Emerging inflammatory response biomarkers are developed to predict the survival of patients with cancer, the aim of our study is to establish an inflammation-related nomogram based on the classical predictive biomarkers to predict the survivals of patients with non-small cell lung cancer (NSCLC).

Methods: Nine hundred and fifty-two NSCLC patients with lung cancer surgery performed were enrolled into this study. The cutoffs of inflammatory response biomarkers were determined by Receiver operating curve (ROC). Univariate and multivariate analysis were conducted to select independent prognostic factors to develop the nomogram.

Results: The median follow-up time was 40.0 months (range, 1 to 92 months). The neutrophil to lymphocyte ratio (cutoff: 3.10, HR:1.648, $P=0.045$ ) was selected to establish the nomogram which could predict the 5 -year OS probability. The C-index of nomogram was 0.72 and the 5 -year OS calibration curve displayed an optimal agreement between the actual observed outcomes and the predictive results.

Conclusions: Neutrophil to lymphocyte ratio was shown to be a valuable biomarker for predicting survival of patients with NSCLC. The addition of neutrophil to lymphocyte ratio could improve the accuracy and predictability of the nomogram in order to provide reference for clinicians to assess patient outcomes.
\end{abstract}

Keywords: Non-small cell lung cancer, Inflammatory response biomarker, Nomogram

\section{Background}

Lung cancer remains the leading cause of cancer-related death worldwide and $85 \%$ of lung cancers diagnosis are non-small cell lung cancer (NSCLC). Numerous studies investigated the prognostic factors in the early stage patients in order to establish a more efficient model to assess patient prognosis. In the seventh edition of the American Joint Committee on Cancer TNM classification, tumor extent, lymph node involvement and distant metastasis contributed significantly to individualized survival predictions [1]. In recent years, more studies reported that tumor characteristics were not the only determinants to predict

\footnotetext{
* Correspondence: liuqi66@sdu.edu.cn; dujiajun@sdu.edu.cn

${ }^{\dagger}$ Ying Wang and Xiao Qu contributed equally to this work.

${ }^{1}$ Institute of Oncology, Shandong Provincial Hospital Affiliated to Shandong

University, 324 Jingwu Road, Jinan 250021, People's Republic of China

Full list of author information is available at the end of the article
}

the outcomes of patients with cancer. As inflammation emerged as a hallmark of cancer, inflammatory response biomarkers have shown to be promising prognostic factors for improving the predictive accuracy in cancer research. In 1986, Shoenfeld et al. demonstrated that high level of white blood cells in peripheral blood was associated with poor outcomes in patients who suffered from non-hematological malignancies [2]. Neutrophil to lymphocyte ratio [3-9], calculated by the ratio of absolute neutrophil counts to absolute lymphocyte counts in whole blood, was established by Walsh et al. who reported its potential prognostic value in colorectal cancer [10]. Additionally, derived neutrophil to lymphocyte ratio $[5,11,12]$, lymphocyte to monocyte ratio [13, 14], platelet to lymphocyte ratio $[3,7]$ and systematic immune-inflammation index [15] were considered as potential systematic inflammatory response biomarkers for survival prediction. 
Although some articles have studied the prognostic or predictive value of these inflammatory response biomarkers, inflammation-related nomogram on NSCLC remains undefined.

Nomogram is a relative novel and convenient model to predict survivals of patients with cancer. It could generate an intuitive graph by integrating diverse determinant variables and reflect an individual probability of a clinical event. Postoperative nomograms can assist patients and physicians to get more information about the prognosis.

In this study, we have evaluated the prognostic values of various inflammatory response biomarkers and selected the most significant factors to establish our nomogram model. The established nomogram was compared with traditional TMN staging system to validate its effectiveness.

\section{Methods}

From January 2006 to December 2011, 1454 patients with lung cancer (including adenocarcinoma or squamous cell carcinoma) who underwent surgery in Shandong Provincial Hospital Affiliated to Shandong University were retrospectively reviewed and consecutively selected. The clinical stages of all patients were identified according to the seventh edition TNM classification. The exclusion criteria included: Patients with incomplete clinical and pathological data; patients with distant metastasis or stage IV; Patients whose primary cancers were not lung cancer; Patients who received radiotherapy or chemotherapy before surgery. We reviewed the hospital records of 952 patients who met the criteria. All patients underwent lung resection and systematic lymph node sampling. Demographic data (age, gender), clinical characteristics (biochemical index, smoking history), histopathological results (pathological type, differentiation, pathological stage of tumor and involved lymph nodes according to TNM system staging), postoperative outcomes and survival data were collected and recorded. Tumor size was assessed using the longest diameter of the tumor. The information of tumor size, nodal metastases and distant metastasis were collected from the pathological and medical image reports.

\section{Ethics statement}

All patients provided written informed consent for their information to be stored in the hospital database and used for research. Ethical approval was obtained from Provincial Hospital Affiliated to Shandong University ethics committee, and the study was carried out in accordance with the approved guidelines.

\section{Postoperative Treatment and Follow-up}

All patients involved in our study were followed up from surgery to July 2014. The minimal follow-up period was 36.0 months (range, 1 to 92 months) and median follow-up time was 40.0 months. Routine examinations such as CT scan postoperatively were performed every 3 months for the first year, every 6 months for the second year and then once a year thereafter.

\section{Candidate biomarkers}

The hematological variables were obtained from blood tests routinely performed 1-3 days before surgery. Inflammatory response biomarkers included: neutrophil to lymphocyte ratio, absolute neutrophil counts to absolute lymphocyte counts, lymphocyte to monocyte ratio, platelet to lymphocyte ratio and systematic immune-inflammation index, which were calculated in the analysis. Neutrophil to lymphocyte ratio is defined as the ratio of absolute neutrophil count to absolute lymphocyte count in whole blood. Absolute neutrophil counts to absolute lymphocyte counts is defined as the ratio of absolute neutrophil count to the absolute white cell count minus the absolute count of neutrophils in whole blood. Platelet to lymphocyte ratio is defined as the ratio of absolute platelet count to absolute lymphocyte count in whole blood. Lymphocyte to monocyte ratio is defined as the ratio of absolute lymphocyte count to the absolute monocyte count in whole blood. Systematic immune-inflammation index is defined as the results of the peripheral platelet count multiplied by neutrophil count and divided by lymphocyte counts in whole blood.

\section{Statistical analysis}

Demographic characteristics were showed through descriptive statistics. Normally distributed continuous data was presented as mean \pm standard deviation, while discrete data was presented as count and proportion. Overall survival (OS) was defined as the period from surgery to death or the last date of follow-up for patients alive. The optimal cut-off levels of neutrophil to lymphocyte ratio, absolute neutrophil counts to absolute lymphocyte counts, lymphocyte to monocyte ratio and platelet to lymphocyte ratio were obtained by ROC analysis based on OS. Survival curves were derived by the Kaplan-Meier method and were assessed by log-rank test univariately. A Cox proportional hazards model was used to conduct multivariate analysis, with a significance level set at two-sided 0.05. Multivariable stepwise Cox models were performed to select final variables for prognostic factors. Above steps were performed with the statistical software SPSS version 20.0. 
Based on the results of the multivariable analysis, a nomogram was established by R 3.2.0 software (Institute for Statistics and Mathematics, Vienna, Austria) with the rms and survival package. Internal validation of the nomogram was conducted and it was subjected to 1000 bootstrap resamples. Then we compared this nomogram with traditional TNM system staging by Harrell's concordance index (c-index) to validate the accuracy of the nomogram. After bias correction, calibration curves on 5-year OS were generated by comparison between the predicted survival and observed survival [16].

\section{Results}

\section{Clinicopathological features}

Totally 952 eligible NSCLC patients, 674 men and 278 women, were enrolled into this study, with a mean age of 59 years (range, 20 to 79 years old). The primary tumor size ranged from 3 to $130.0 \mathrm{~mm}$ with a mean size of $38.6 \mathrm{~mm}$, while the pathologic $\mathrm{T}$ stage showed 300 patients were in pathologic T1, 515 in pathologic T2,79 in pathologic T3 and 58 in pathologic T4. According to TNM system staging, pathological $\mathrm{N}$ stages were divided into three levels, and among them there were 530 pathologic NO patients, 204 pathologic N1 patients, 213 pathologic N2 patients and 5 pathologic N3 patients. There were 416 patients with squamous cell carcinoma and 536 patients with adenocarcinoma respectively. Regarding degree of tumor differentiation, 131 patients were identified as well differentiated, 676 patients were identified as moderately differentiated and 145 patients were identified as poorly differentiated. Among the enrolled patients, 772 patients had the smoking experience and 180 patients did not have the experience. There were 483 patients received adjuvant chemotherapy after surgery and 483 patients did not receive chemotherapy. The characteristic information based on neutrophil to lymphocyte ratio was shown in Table 1. The optimal cut-offs obtained from ROC curves of neutrophil to lymphocyte ratio, absolute neutrophil counts to absolute lymphocyte counts, lymphocyte to monocyte ratio and platelet to lymphocyte ratio and systematic immune-inflammation index were shown in Table 2. Patients were divided into groups on the basis of optimal cut-offs.

Independent prognostic factors screened for nomogram Kaplan-Meier survival analysis was conducted to evaluate the relationship between inflammatory response biomarkers and survival outcomes. Patients were divided into two groups based on the optimal cutoffs of inflammatory response biomarkers (in Table 3), and all groups
Table 1 The clinicopathological characteristics based on neutrophil to lymphocyte ratio

\begin{tabular}{|c|c|c|c|}
\hline & Total & NLR & \\
\hline & & $<3.1(n=732)$ & $>3.1(n=220)$ \\
\hline Gender & & & \\
\hline Male & 674 & 486 & 188 \\
\hline Female & 278 & 246 & 32 \\
\hline Age & $59(20-79)$ & $59(20-79)$ & $60(27-78)$ \\
\hline Smoking $\mathrm{h}$ & & & \\
\hline $\mathrm{N}$ & 180 & 126 & 54 \\
\hline Y & 772 & 606 & 166 \\
\hline pT categor & & & \\
\hline pT1 & 300 & 233 & 67 \\
\hline pT2 & 515 & 391 & 124 \\
\hline pT3 & 79 & 61 & 18 \\
\hline pT4 & 58 & 47 & 11 \\
\hline pN catego & & & \\
\hline $\mathrm{pNO}$ & 530 & 416 & 114 \\
\hline $\mathrm{pN} 1$ & 204 & 150 & 54 \\
\hline $\mathrm{pN} 2$ & 213 & 163 & 50 \\
\hline pN3 & 5 & 3 & 2 \\
\hline Histology & & & \\
\hline$A D C$ & 536 & 453 & 83 \\
\hline SCC & 416 & 279 & 137 \\
\hline PGTD & & & \\
\hline I & 131 & 111 & 20 \\
\hline$\|$ & 676 & 508 & 168 \\
\hline III & 145 & 113 & 32 \\
\hline Chemothe & & & \\
\hline $\mathrm{N}$ & 469 & 371 & 98 \\
\hline Y & 483 & 361 & 122 \\
\hline
\end{tabular}

pT category pathologcial T category $p N$ category pathologcial $\mathrm{N}$ category $A D C$ adenocarcinoma

SCC squamous cell carcinoma

PGTD pathological grading of tumor differentiation

NLR neutrophil to lymphocyte ratio

Table 2 The optimal cut-off point based on OS

\begin{tabular}{lllll}
\hline & Median values & Range & AUC & Cut-off \\
\hline NLR & 2.49 & $0.33-12.40$ & 0.584 & 3.1 \\
dNLR & 0.68 & $0.21-9.79$ & 0.423 & 0.499 \\
PLR & 140.43 & $31.22-450.00$ & 0.553 & 170.58 \\
LMR & 4.72 & $0.66-195.00$ & 0.428 & 3.53 \\
SII & 614.99 & $76.26-3954.03$ & 0.582 & 781.82 \\
\hline
\end{tabular}

NLR neutrophil to lymphocyte ratio

$d N L R$ derived neutrophil to lymphocyte ratio

$P L R$ platelet to lymphocyte ratio

$L M R$ lymphocyte to monocyte ratio

SIl systematic immune-inflammation index 
Table 3 Univariable analysis and cox proportional hazards regression analysis

\begin{tabular}{|c|c|c|c|c|c|c|}
\hline \multirow[t]{2}{*}{ Variable } & \multicolumn{3}{|c|}{ Univariable analysis } & \multicolumn{3}{|c|}{ Multivariable analysis } \\
\hline & Hazard ratio & $95 \% \mathrm{Cl}$ & $P$ & Hazard ratio & $95 \% \mathrm{Cl}$ & $P$ \\
\hline Age & 1.388 & $1.112-1.733$ & 0.004 & 1.649 & $1.306-2.081$ & $<0.001$ \\
\hline \multicolumn{7}{|l|}{ Gender } \\
\hline Female & $R$ & & & & & \\
\hline Male & 1.243 & $0.965-1.601$ & 0.092 & & & \\
\hline \multicolumn{7}{|l|}{ Smoking history } \\
\hline N & $R$ & & & & & \\
\hline Y & 1.426 & $1.120-1.815$ & 0.004 & 1.157 & $0.878-1.524$ & 0.300 \\
\hline \multicolumn{7}{|l|}{ pT category } \\
\hline $\mathrm{T} 1-2$ & R & & & $\mathrm{R}$ & & 0.007 \\
\hline T3-4 & 2.030 & $1.558-2.645$ & $<0.001$ & 1.455 & $1.097-1.930$ & 0.009 \\
\hline \multicolumn{7}{|l|}{ pN category } \\
\hline No & R & & $<0.001$ & $\mathrm{R}$ & & $<0.001$ \\
\hline N1 & 2.414 & $1.808-3.22$ & $<0.001$ & 2.277 & $1.690-3.067$ & $<0.001$ \\
\hline N2 & 4.097 & $3.153-5.323$ & $<0.001$ & 4.233 & $3.216-5.570$ & $<0.001$ \\
\hline N3 & 6.170 & $1.953-19.493$ & 0.002 & 5.121 & $1.530-17.144$ & 0.008 \\
\hline \multicolumn{7}{|l|}{ Histology } \\
\hline Histology ADC & R & & & R & & \\
\hline Histology CC & 1.311 & $1.050-1.636$ & 0.017 & 0.965 & $0.744-1.252$ & 0.789 \\
\hline \multicolumn{7}{|l|}{ PGTD } \\
\hline PGTD I & R & & $<0.001$ & R & & 0.008 \\
\hline PGTD ॥ & 0.174 & $0.091-0.332$ & $<0.001$ & 2.671 & $1.433-4.979$ & 0.002 \\
\hline PGTD ॥ & 0.789 & $0.594-1.049$ & 0.103 & 2.563 & $1.315-4.999$ & 0.006 \\
\hline \multicolumn{7}{|l|}{ Chemotherapy } \\
\hline N & $\mathrm{R}$ & & & & & \\
\hline Y & 1.166 & $0.934-1.457$ & 0.175 & & & \\
\hline \multicolumn{7}{|l|}{ NLR } \\
\hline$<3.1$ & $R$ & & & & & \\
\hline$>3.1$ & 1.845 & $1.457-2.337$ & $<0.001$ & 1.648 & $1.010-2.687$ & 0.045 \\
\hline \multicolumn{7}{|l|}{ dNLR } \\
\hline$<0.499$ & $\mathrm{R}$ & & & & & \\
\hline$>0.499$ & 0.627 & $0.497-0.790$ & $<0.001$ & 1.470 & $0.936-2.309$ & 0.095 \\
\hline \multicolumn{7}{|l|}{ PLR } \\
\hline$<170.58$ & $\mathrm{R}$ & & & & & \\
\hline$>170.58$ & 1.636 & $1.284-2.085$ & $<0.001$ & 1.201 & $0.895-1.621$ & 0.221 \\
\hline \multicolumn{7}{|l|}{ LMR } \\
\hline$<3.53$ & $R$ & & & & & \\
\hline$>3.53$ & 0.619 & $0.496-0.772$ & $<0.001$ & 1.020 & $0.770-1.351$ & 0.890 \\
\hline \multicolumn{7}{|l|}{ SII } \\
\hline$<781.82$ & R & & & & & \\
\hline$>781.82$ & 1.852 & $1.463-2.344$ & $<0.001$ & 1.412 & $0.955-2.090$ & 0.084 \\
\hline
\end{tabular}

pT category pathologcial T category

$p N$ category pathologcial $\mathrm{N}$ category

$R$ reference

$A D C$ adenocarcinoma

SCC squamous cell carcinoma

PGTD pathological grading of tumor differentiation

$N L R$ neutrophil to lymphocyte ratio

$d N L R$ derived neutrophil to lymphocyte ratio

$P L R$ platelet to lymphocyte ratio

$L M R$ lymphocyte to monocyte ratio

SIl systematic immune-inflammation index 

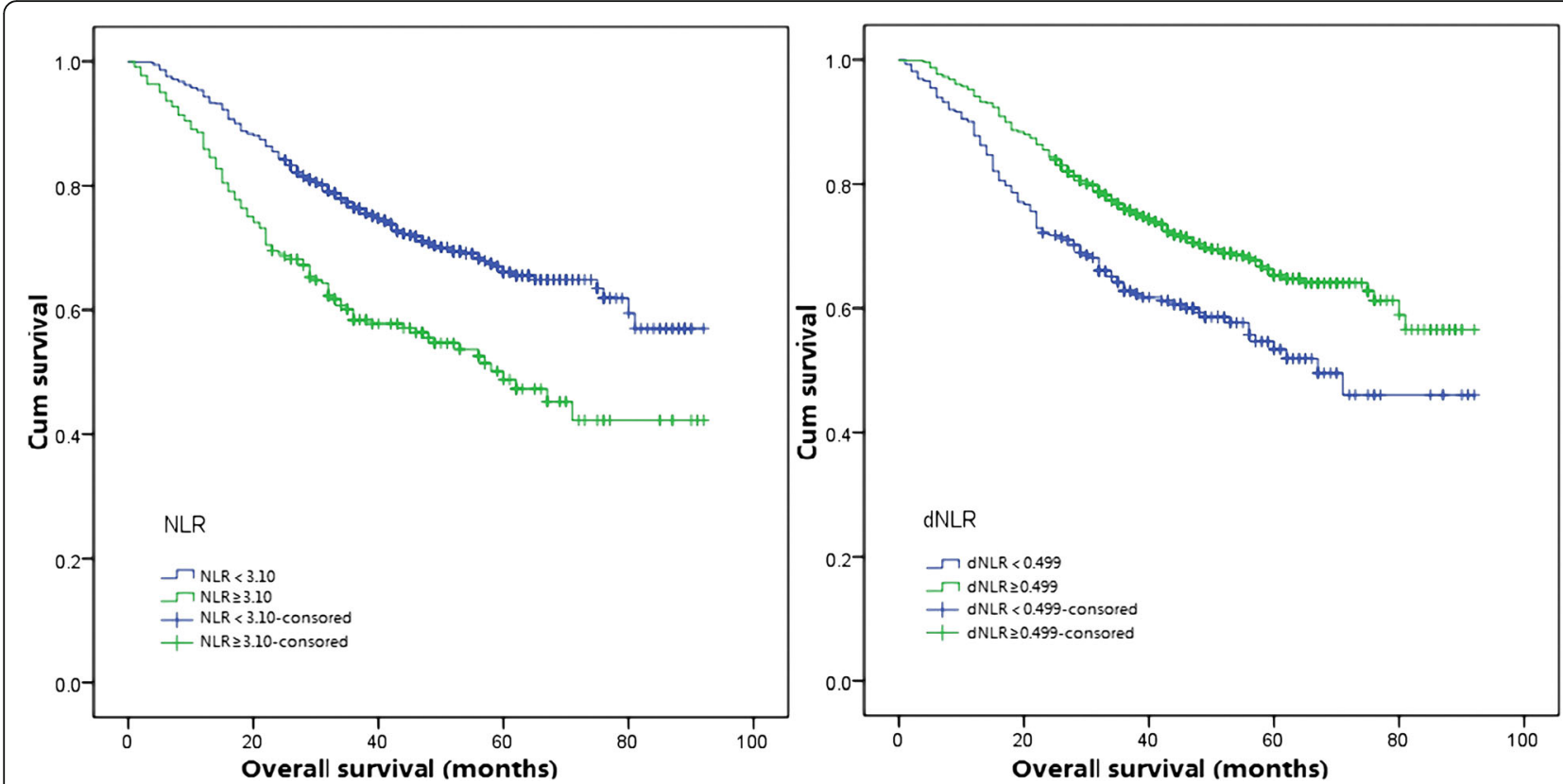

Fig. 1 Kaplan-Meier curves for overall survival according to NLR and dNLR. NLR: Neutrophil to lymphocyte ratio; dNLR: Derived neutrophil to lymphocyte ratio

had significantly different survival ends(in Figs. 1 and 2). The univariate analysis indicated that age, neutrophil to lymphocyte ratio, absolute neutrophil counts to absolute lymphocyte counts, lymphocyte to monocyte ratio and platelet to lymphocyte ratio and systematic immune-inflammation index, pathologic $\mathrm{T}$ staging, pathologic $\mathrm{N}$ staging, tumor differentiation and smoking history were associated with OS (in Table 4). Multivariate analysis suggested that age, pathologic $\mathrm{T}$ and $\mathrm{N}$ staging, tumor differentiation, neutrophil to lymphocyte ratio were significantly associated with patients with reduced OS.

\section{Prognostic nomogram on OS}

A nomogram was established which embraced the significant prognostic factors, age, pathologic $\mathrm{T}$ and $\mathrm{N}$ staging, tumor differentiation, and neutrophil to lymphocyte ratio and had the ability to reflect the 5-year OS (in Fig. 3). The nomogram evinced that neutrophil to lymphocyte ratio made a significant contribution to survival outcomes.

\section{Internal validation and calibration plot}

The C-index was 0.72 in the nomogram, higher than that of TNM system staging (0.69). Afterwards, the 5 -year OS calibration curves of our nomogram displayed an optimal agreement between the actual observed outcomes and the predictions (in Fig. 4), compared with
TNM system staging. The nomogram of our model was validated by the sample size of 100 , while TNM system staging was validated by the sample size of 300 for its fewer variates. In the same time, The ROC of the nomogram was performed and the AUC of our nomogram was 0.767 (Fig. 5).

\section{Discussion}

Although there have been several nomograms used to select individual therapy for patients with lung cancer $[16,17]$, a nomogram incorporated with inflammatory response biomarkers has not been put forward. The aim of our study is to investigate the impact of inflammatory response biomarkers on survival outcomes and to establish an inflammation-related nomogram in patients with NSCLC who underwent surgery.

In our study, both classical and novel inflammatory response biomarkers are the candidates for nomogram including: neutrophil to lymphocyte ratio, absolute neutrophil counts to absolute lymphocyte counts, lymphocyte to monocyte ratio and platelet to lymphocyte ratio and systematic immune-inflammation index. All the biomarkers show their predictive ability on survival outcomes and among them, only the neutrophil to lymphocyte ratio has been selected to be included in the nomogram after survival analysis through Kaplan-Meier curves, univariate and multivariate method. For 

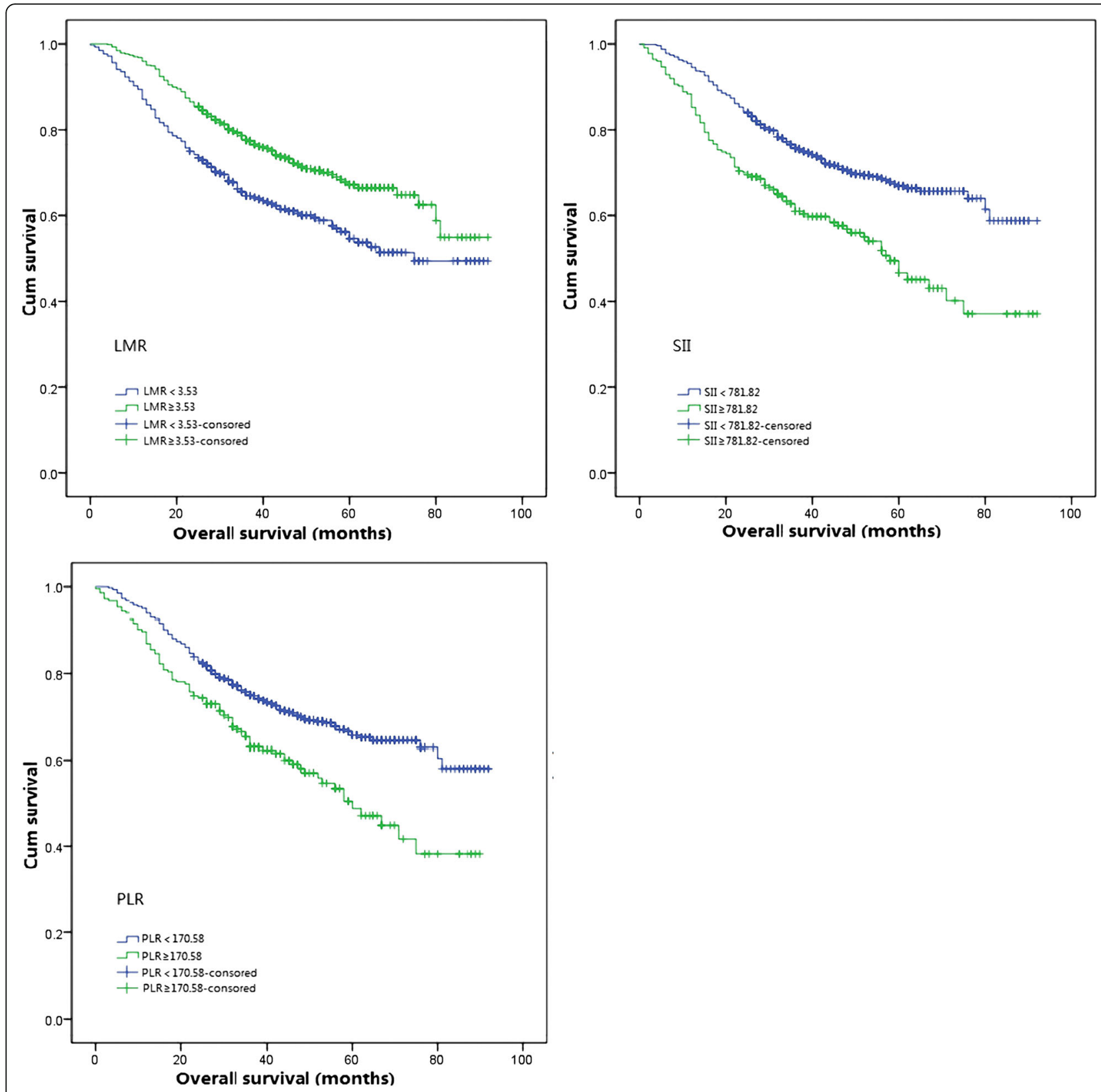

Fig. 2 Kaplan-Meier curves for overall survival according to SII, LMR and PLR. PLR: platelet to lymphocyte ratio; LMR: lymphocyte to monocyte ratio; SII: systematic immune-inflammation index

non-inflammatory biomarkers, pathologic $\mathrm{T}$ and $\mathrm{N}$ staging, age and tumor differentiation are also considered as independent prognostic factors which could be incorporated into the nomogram. In the nomogram, neutrophil to lymphocyte ratio is the third most important prognostic factors following pathologic $\mathrm{N}$ staging and age to predict the survival. Internal validation and calibration curve are performed to test the repeatability and reliability of the nomogram. Compared with TNM traditional system staging, the nomogram has a higher C-index (0.72) through internal validation, indicating that the nomogram has a better ability to discriminate survival outcomes. Calibration curves for the nomogram of 5-year OS disclose an excellent agreement between prediction and actual observation and is superior to those of TNM system staging. Based on the above results, we believe that inflammatory response biomarkers should be incorporated into the predictive models as independent prognostic factors of patients with lung cancer, and the inflammation-related nomogram have been shown to provide more precise prediction compared with traditional TNM classification. 
Table 4 The survival data of subgroups according to inflammation response biomarkers

\begin{tabular}{|c|c|c|c|c|c|}
\hline Groups & Cutoff & Patients & 3-year OS & 5-year OS & P \\
\hline NLR & & & & & $<0.001$ \\
\hline$<3.10$ & & 732 & $76.50 \%$ & $66.10 \%$ & \\
\hline$>3.10$ & & 220 & $58.40 \%$ & $48.80 \%$ & \\
\hline$d N L R$ & & & & & $<0.001$ \\
\hline$<0.499$ & & 262 & $79.50 \%$ & $62.80 \%$ & \\
\hline$>0.499$ & & 690 & $65.30 \%$ & $53.40 \%$ & \\
\hline PLR & & & & & $<0.001$ \\
\hline$<170.58$ & & 738 & $75.00 \%$ & $63.00 \%$ & \\
\hline$>170.58$ & & 214 & $65.70 \%$ & $48.80 \%$ & \\
\hline LMR & & & & & $<0.001$ \\
\hline$<3.53$ & & 388 & $64.60 \%$ & $77.60 \%$ & \\
\hline$>3.53$ & & 564 & $54.60 \%$ & $57.20 \%$ & \\
\hline SII & & & & & $<0.001$ \\
\hline$<781.82$ & & 729 & $75.70 \%$ & $61.00 \%$ & \\
\hline$>781.82$ & & 233 & $66.90 \%$ & $46.70 \%$ & \\
\hline
\end{tabular}

$N L R$ neutrophil to lymphocyte ratio

$d N L R$ derived neutrophil to lymphocyte ratio

$P L R$ platelet to lymphocyte ratio

LMR lymphocyte to monocyte ratio

SIl systematic immune-inflammation index

Cancer-related inflammation has been referred as local inflammation and systemic inflammation which could promote tumorigenesis and metastasis [18] in a broad range of cancers [19]. Increasing novel inflammatory response biomarkers are therefore developed to better refine the stratification of patients. Recently, increasing attention is being paid to the biomarkers derived from innate immune cells in peripheral blood. Neutrophil to lymphocyte ratio is a simple index of the systemic inflammatory response, and the increased level of neutrophil to lymphocyte ratio has been shown to predict worse overall survival in patients with NSCLC [3, 4, 6-8]. Additionally, it has been reported that the perioperative use of nonsteroidal anti-inflammatory drugs (NSAIDs), such as celecoxib and ketorolac, could change the tumor microenvironment and reduce migration and invasion of circulating malignant cells [4, 20-22]. Taken together, these findings demonstrate the importance of perioperative inflammation and immune suppression on oncological outcomes.

Neutrophils could be stimulated to proliferate by cancer-related inflammatory factors, such as Tumor necrosis factor-alpha and Interleukin-6, which subsequently secrete reactive oxygen species and pro-angiogenic factors, and therefore favors tumorigenesis and tumor microenvironment [23, 24]. Also, bone marrow could lead to an abnormal release of neutrophils precursors upon inflammation. Regarding lymphocytes, they have shown to exert a vital role in cell-mediated immunity against host cancer cells, and the decreases in lymphocytes count have worse survival outcomes Nomograms possess their own merits of predicting oncologic prognosis, such as intuitive graphs and numerical probability of clinical events, so they are identified as reliable tools to quantify risks. Given the importance of neutrophils and lymphocytes in tumor development, we therefore seek to integrate the neutrophil to lymphocyte ratio into the nomogram for improving the accuracy of the predictive model. Our results indicate that the contribution of neutrophil to lymphocyte ratio

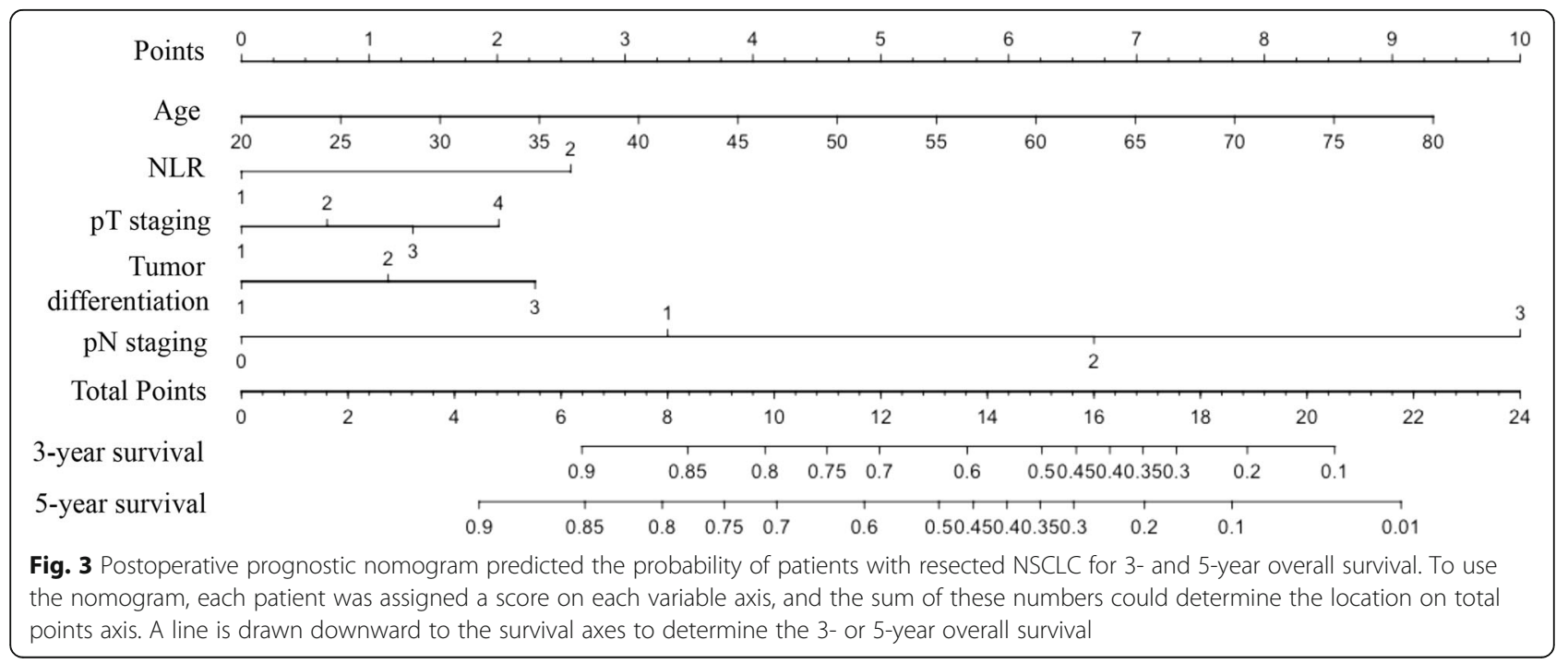



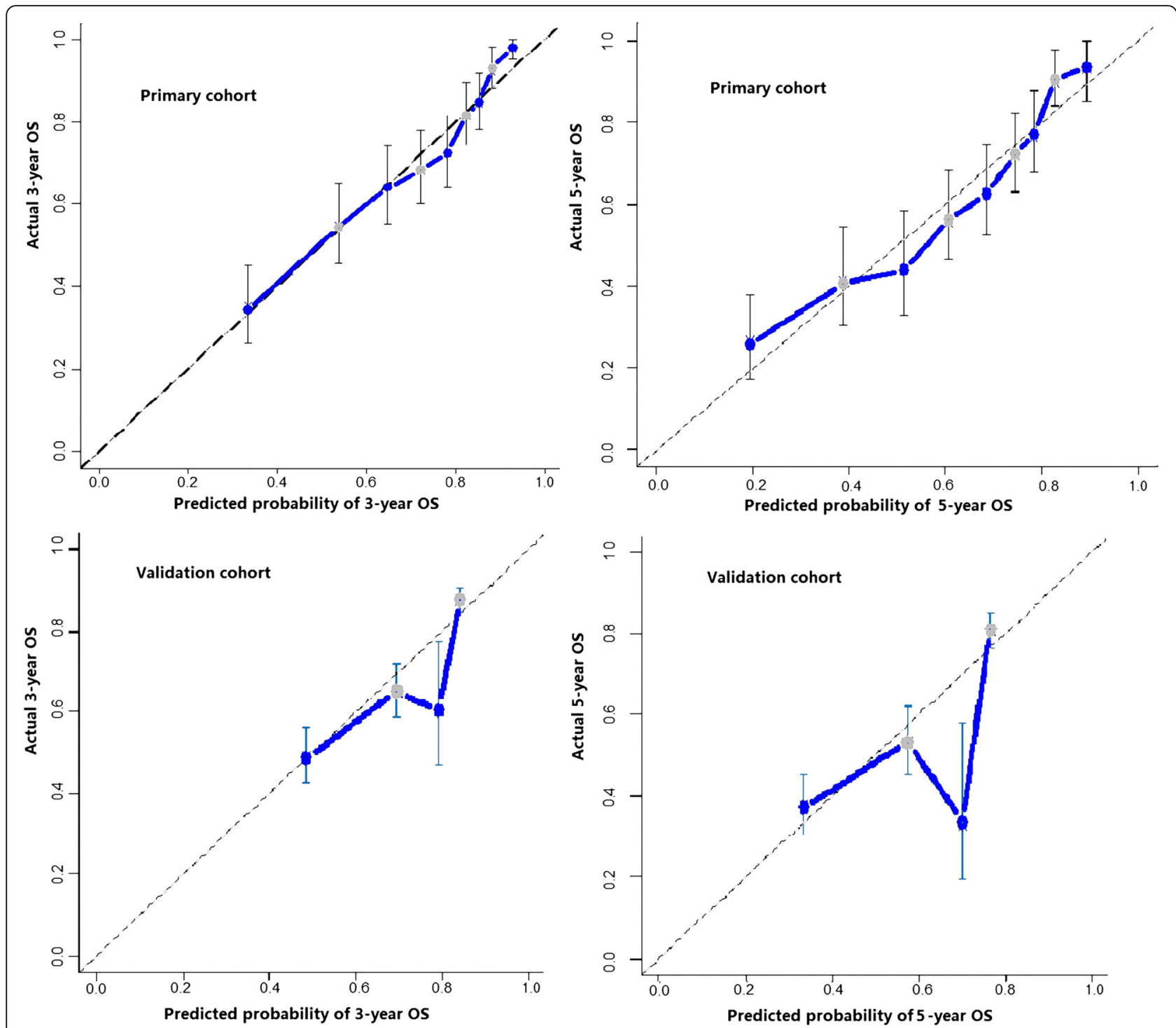

Fig. 4 The calibration curves for predicting patient survival of 3- and 5-year OS in the primary cohort and Validation cohort

is at the third place as a predictor, following pathologic $\mathrm{N}$ staging and age. Our proposed nomogram highlights the significant predictive role of neutrophil to lymphocyte ratio in prognosis.

Apart from inflammatory response biomarkers, age, tumor differentiation, pathologic $\mathrm{T}$ stage and pathologic $\mathrm{N}$ stage are the other independent prognostic factors which reveal a significant influence on survival. The nomogram incorporated with neutrophil to lymphocyte ratio might have the ability to predict the prognosis of patients undergoing surgery according to their inflammatory status, pathologic $\mathrm{T}$ and pathologic $\mathrm{N}$ stages and other tumor characteristics. Moreover, our nomogram could also assist clinicians in developing tailored treatment for individual patients based on their inflammatory status.

Our nomogram has some limitations. First, the analysis is conducted retrospectively which creates intrinsic drawbacks. Second, some prognostic parameters (such as carcinoembryonic levels) and other important molecular factors (such as Epithelial growth factor receptor mutation) are not included in our analysis due to lack of data.

\section{Conclusions}

In conclusion, we have established an inflammation-related prognostic nomogram predicting individual survival in patients with NSCLC after surgery. Additionally, neutrophil to lymphocyte ratio can be considered as an 


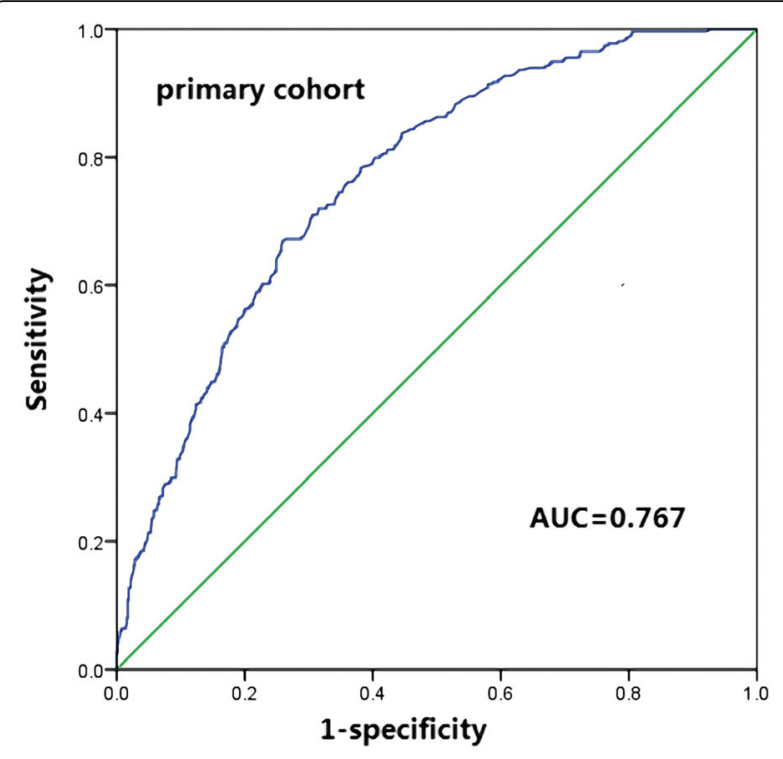

Fig. 5 The AUC of the nomogram

independent prognostic factor. The proposed nomogram in this study provides better predictive accuracy and confirms the predictive value of inflammation response biomarkers. It offers a useful tool for providing reference for clinicians to assess the survival of individual patients after surgery.

\section{Abbreviations \\ ADC: Adenocarcinoma; dNLR: Derived neutrophil to lymphocyte ratio; LMR: Lymphocyte to monocyte ratio; NLR: Neutrophil to lymphocyte ratio; NSAID: Nonsteroidal anti-inflammatory drug; NSCLC: Non-small cell lung cancer; OS: Overall survival; PGTD: Pathological grading of tumor differentiation; PLR: Platelet to lymphocyte ratio; pN category: pathologcial N category; PT category: pathologcial T category; R: Reference; ROC: Receiver operating curve; SCC: Squamous cell carcinoma; SII: Systematic immune- inflammation index}

\section{Funding}

The work was supported by National Natural Science Foundation of China (81301728) and Provincial Natural Science Foundation of Shandong (ZR2013HZ001) and (ZR2014HM100). The funding body had no role in the design of the study and collection, analysis, and interpretation of data and in writing the manuscript.

\section{Availability of data and materials}

The datasets generated and analyzed during the current study are not publicly available due to it is a part of Shandong Provincial Hospital database but are available from the corresponding author on reasonable request.

\section{Authors' contributions}

JJD and QL have full access to all of the data in the study and take responsibility for the integrity of the data and the accuracy of the data analysis. YW and XQ: contributed to the study design, definition of the inclusion and exclusion criteria, data analysis and interpretation, and drafting and revision the manuscript. NWK, HCS, KW: contributed to the study design and revision the manuscript. All the authors read and approved the final manuscript.

\section{Ethics approval and consent to participate}

All patients gave written informed consent for their information to be stored in the hospital database and used for research. Ethical approval was obtained from Provincial Hospital Affiliated to Shandong University ethics committee, and the study was carried out in accordance with the approved guidelines.

\section{Competing interests}

The authors declare that they have no competing interests.

\section{Publisher's Note}

Springer Nature remains neutral with regard to jurisdictional claims in published maps and institutional affiliations.

\section{Author details}

'Institute of Oncology, Shandong Provincial Hospital Affiliated to Shandong University, 324 Jingwu Road, Jinan 250021, People's Republic of China.

${ }^{2}$ Department of Thoracic Surgery, Shandong Provincial Hospital Affiliated to Shandong University, 324 Jingwu Road, Jinan 250021, People's Republic of China. ${ }^{3}$ Department of Oncology, Shandong Provincial Hospital Affiliated to Shandong University, 324 Jingwu Road, Jinan 250021, People's Republic of China. ${ }^{4}$ Department of Clinical Oncology, The University of Hong Kong, Laboratory block, 21 Sassoon, Pokfulam, Hong Kong, People's Republic of China.

Received: 3 July 2017 Accepted: 16 May 2018

Published online: 26 June 2018

\section{References}

1. Groome PA, Bolejack V, Crowley JJ, Kennedy C, Krasnik M, Sobin LH, Goldstraw P. The IASLC lung Cancer staging project: validation of the proposals for revision of the $\mathrm{T}, \mathrm{N}$, and $\mathrm{M}$ descriptors and consequent stage groupings in the forthcoming (seventh) edition of the TNM classification of malignant tumours. J Thorac Oncol. 2007;2(8):694-705.

2. Shoenfeld Y, Tal A, Berliner S, Pinkhas J. Leukocytosis in non hematological malignancies-a possible tumor-associated marker. J Cancer Res Clin Oncol. 1986;111(1):54-8.

3. Cannon NA, Meyer J, lyengar P, Ahn C, Westover KD, Choy H, Timmerman R. Neutrophil-lymphocyte and platelet-lymphocyte ratios as prognostic factors after stereotactic radiation therapy for early-stage non-small-cell lung cancer. J Thorac Oncol. 2015;10(2):280-5.

4. Choi JE, Villarreal J, Lasala J, Gottumukkala V, Mehran RJ, Rice D, Yu J, Feng L, Cata JP. Perioperative neutrophil:lymphocyte ratio and postoperative NSAID use as predictors of survival after lung cancer surgery: a retrospective study. Cancer Med. 2015;4(6):825-33.

5. Dirican A, Kucukzeybek BB, Alacacioglu A, Kucukzeybek Y, Erten C, Varol U, Somali I, Demir L, Bayoglu IV, Yildiz Y, et al. Do the derived neutrophil to lymphocyte ratio and the neutrophil to lymphocyte ratio predict prognosis in breast cancer? Int J Clin Oncol. 2015;20(1):70-81.

6. Huang C, Yue J, Li Z, Li N, Zhao J, Qi D. Usefulness of the neutrophil-tolymphocyte ratio in predicting lymph node metastasis in patients with nonsmall cell lung cancer. Tumour Biol. 2015;36(10):7581-9.

7. Kemal Y, Yucel I, Ekiz K, Demirag G, Yilmaz B, Teker F, Ozdemir M. Elevated serum neutrophil to lymphocyte and platelet to lymphocyte ratios could be useful in lung cancer diagnosis. Asian Pac J Cancer Prev. 2014;15(6):2651-4.

8. Lin GN, Peng JW, Liu PP, Liu DY, Xiao JJ, Chen XQ. Elevated neutrophil-tolymphocyte ratio predicts poor outcome in patients with advanced nonsmall-cell lung cancer receiving first-line gefitinib or erlotinib treatment. Asia-Pacific journal of clinical oncology 2017;13(5):e189-e194.

9. Sarraf KM, Belcher E, Raevsky E, Nicholson AG, Goldstraw P, Lim E. Neutrophil/lymphocyte ratio and its association with survival after complete resection in non-small cell lung cancer. J Thorac Cardiovasc Surg. 2009; 137(2):425-8.

10. Walsh SR, Cook EJ, Goulder F, Justin TA, Keeling NJ. Neutrophillymphocyte ratio as a prognostic factor in colorectal cancer. J Surg Oncol. 2005;91(3):181-4.

11. Absenger G, Szkandera J, Pichler M, Stotz M, Arminger F, Weissmueller M, Schaberl-Moser R, Samonigg H, Stojakovic T, Gerger A. A derived neutrophil to lymphocyte ratio predicts clinical outcome in stage II and III colon cancer patients. Br J Cancer. 2013;109(2):395-400.

12. Szkandera J, Gerger A, Liegl-Atzwanger B, Stotz M, Samonigg H, Friesenbichler J, Stojakovic T, Leithner A, Pichler M. The derived neutrophil/ lymphocyte ratio predicts poor clinical outcome in soft tissue sarcoma patients. Am J Surg. 2014;210(1):111-6. 
13. Huang Y, Feng JF. Low preoperative lymphocyte to monocyte ratio predicts poor cancer-specific survival in patients with esophageal squamous cell carcinoma. OncoTargets Ther. 2015;8:137-45.

14. Lin GN, Peng JW, Xiao JJ, Liu DY, Xia ZJ. Prognostic impact of circulating monocytes and lymphocyte-to-monocyte ratio on previously untreated metastatic non-small cell lung cancer patients receiving platinum-based doublet. Med Oncol. 2014;31(7):70.

15. Hu B, Yang XR, Xu Y, Sun YF, Sun C, Guo W, Zhang X, Wang WM, Qiu SJ, Zhou J, et al. Systemic immune-inflammation index predicts prognosis of patients after curative resection for hepatocellular carcinoma. Clin Cancer Res. 2014;20(23):6212-22.

16. Liang W, Zhang L, Jiang G, Wang Q, Liu L, Liu D, Wang Z, Zhu Z, Deng Q, Xiong $X$, et al. Development and validation of a nomogram for predicting survival in patients with resected non-small-cell lung cancer. J Clin Oncol. 2015:33(8):861-9.

17. Keam B, Kim DW, Park JH, Lee JO, Kim TM, Lee SH, Chung DH, Heo DS. Nomogram predicting clinical outcomes in non-small cell lung Cancer patients treated with epidermal growth factor receptor tyrosine kinase inhibitors. Cancer Res Treat. 2014;46(4):323-30.

18. Hu P, Shen H, Wang G, Zhang P, Liu Q, Du J. Prognostic significance of systemic inflammation-based lymphocyte- monocyte ratio in patients with lung cancer: based on a large cohort study. PLoS One. 2014;9(9):e108062.

19. Qu X, Pang Z, Yi W, Wang Y, Wang K, Liu Q, Du J. High percentage of alpha1-globulin in serum protein is associated with unfavorable prognosis in non-small cell lung cancer. Med Oncol. 2014;31(10):238.

20. Yuan D, Zhu K, Li K, Yan R, Jia Y, Dang C. The preoperative neutrophillymphocyte ratio predicts recurrence and survival among patients undergoing R0 resections of adenocarcinomas of the esophagogastric junction. J Surg Oncol. 2014;110(3):333-40

21. Forget $P$, Machiels JP, Coulie PG, Berliere M, Poncelet AJ, Tombal B, Stainier A, Legrand C, Canon JL, Kremer Y, et al. Neutrophil:lymphocyte ratio and intraoperative use of ketorolac or diclofenac are prognostic factors in different cohorts of patients undergoing breast, lung, and kidney cancer surgery. Ann Surg Oncol. 2013;20(Suppl 3):S650-60.

22. Zhang S, Da L, Yang X, Feng D, Yin R, Li M, Zhang Z, Jiang F, Xu L. Celecoxib potentially inhibits metastasis of lung cancer promoted by surgery in mice, via suppression of the PGE2-modulated beta-catenin pathway. Toxicol Lett. 2014;225(2):201-7.

23. Kusumanto YH, Dam WA, Hospers GA, Meijer C, Mulder NH. Platelets and granulocytes, in particular the neutrophils, form important compartments for circulating vascular endothelial growth factor. Angiogenesis. 2003;6(4):283-7.

24. McGuire L, Kiecolt-Glaser JK, Glaser R. Depressive symptoms and lymphocyte proliferation in older adults. J Abnorm Psychol. 2002;111(1):192-7.

\section{Ready to submit your research? Choose BMC and benefit from:}

- fast, convenient online submission

- thorough peer review by experienced researchers in your field

- rapid publication on acceptance

- support for research data, including large and complex data types

- gold Open Access which fosters wider collaboration and increased citations

- maximum visibility for your research: over $100 \mathrm{M}$ website views per year 